\title{
Cardiovascular disease in oncology
}

\author{
Bernard A Mason, MD
}

$\mathrm{I}$

n the first quarter of 2015, a number of papers that addressed cardiovascular issues in oncology were published. These included studies of the cardiovascular toxicity of cancer therapy, treatment guidelines from the American Society of Clinical Oncology (ASCO) for venous thromboembolism prophylaxis (VTE), and the prognostic significance of superficial vein thrombosis.

\section{Cardiovascular disease after androgen- deprivation therapy prostate cancer ${ }^{1,2}$}

In this study, based on health care registers in Sweden, the risk of cardiovascular disease (CVD) was analyzed in more than 41,000 men with prostate cancer who had been treated with gonadotropin-releasing hormone (GnRH ) agonists, surgical orchiectomy, or antiandrogens from 2006-2012.

The following findings on the development of CVD emerged from this large study (all that apply):

a) Men who already had a history of CVD were not at higher risk for the subsequent development of CVD.

b) Men who were treated with orchiectomy, a $\mathrm{GnRH}$ agonist, and antiandrogens had an equal increase in the risk of CVD.

c) The risk for CVD peaked in the first 6 months of treatment.

d) The results of this study are consistent with testosterone being cardioprotective.

Key points

In 2010, the US Food and Drug Administration (FDA) issued a safety warning for $\mathrm{GnRH}$ agonists indicating that this class of drugs could cause an increased risk of diabetes and certain cardiovascular diseases such as heart attack, sudden cardiac death, and stroke in men who were receiving them as treatment for prostate cancer. For many years, androgen deficiency has been known to lead to a loss of muscle mass and an increase in fat resulting in the metabolic syndrome that can lead to diabetes, atherosclerosis, and CVD. The drugs can also result in loss of energy and muscle mass, low bone density, and sexual dysfunction. However, analyses of data from randomized clinical trials of androgen deprivation ther-

apy for the treatment of prostate cancer have yielded conflicting results in regard to CVD risk.

In this study, men who received $\mathrm{GnRH}$ agonist therapy or surgical orchiectomy had an increase in CVD of $21 \%$ and $16 \%$, respectively. However, those receiving antiandrogens were at a decreased risk for CVD (hazard ratio, .87). This implies that the severe deficiency of testosterone was the root cause of CVD. Men receiving antiandrogen therapy have an increased level of testosterone. Those men in the study who had a significant previous history of CVD were at a much higher risk for subsequent CVD within the first 6-12 months of starting androgen deprivation therapy. Among the theories proposed by the authors to explain the findings consistent with these data was that testosterone is a cardioprotective agent.

The findings of this study bring into focus the harm that the hormonal therapy of prostate cancer can cause. In this era of re-evaluating use of the prostate-specific antigen test for the mass screening of men who are at average risk for prostate cancer and the concerns about over-diagnosis and overtreatment, the significant increase in CVD caused by androgen-deprivation therapy is yet another cause for concern.

\section{Answers c, d}

\section{Cerebral small vessel disease in breast cancer survivors treated with adjuvant therapy ${ }^{3}$}

In this study from the Netherlands, 187 breast cancer survivors who had been treated with adjuvant chemotherapy plus radiation more than 20 years previously were compared with 374 age-matched controls who had never had cancer. All of the participants had brain magnetic-resonance imaging (MRI) scans, which were rated for white matter lesion (WML) volume, brain infarctions, and cerebral microbleeds (CMBs).

The results of this study included:

a) WML volume, CMBs, and brain infarctions were more prevalent in the breast cancer survivors.

b) Radiation therapy that included the carotid artery (supraclavicular irradiation) increased the

JCSO 2015;13:231-234. @2015 Frontline Medical Communications. DOI 10.12788/icso.0144. 
incidence of CMBs.

c) A diagnosis of breast cancer alone without any application of adjuvant therapy increased the risk of CMBs.

d) Patients with cerebral microbleeds in deep or infratentorial regions of the brain performed worse on tests of verbal memory and processing speed.

\section{Key points}

The patients selected for this study were all female with unilateral invasive breast cancer who had survived at least 20 years and had been treated with both adjuvant CMF (cyclophosphamide, methotrexate, and fluorouracil) chemotherapy plus radiation therapy. The radiation therapy had been administered to axilla, the breast or chest wall, and for some, supraclavicular with or without internal mammary chain irradiation. Supraclavicular radiation was considered to produce a moderate radiation exposure to the carotid artery. Theoretically, carotid artery irradiation could cause carotid atherosclerosis, which could result in cerebrovascular disease. Therefore, the participants were analyzed according to the type of radiation they had received. The authors gave no information about any form of adjuvant endocrine therapy, so the results did not shed light on any effect of endocrine adjuvant therapy.

The study results indicated that infratentorial CMBs were more frequent in the treated breast cancer patients compared with the controls, but the same did not apply for WML volume or brain infarctions. There was no association with radiation exposure of the carotid artery and WML volume, CMBs, or stroke. This study was not designed to show that a diagnosis of breast cancer by itself increases the risk of CMBs.

The relationship between CMBs and cognitive function was evaluated by testing verbal memory, executive functioning, word fluency, processing speed, visual spatial ability, and manual dexterity. There was a significant relationship between CMBs in deep or infratentorial regions of the brain and a loss of processing speed and memory functioning.

Although future clinical and experimental studies should provide further understanding of the effects of adjuvant chemotherapy on the brain, the findings from this study provide a note of caution about an important potential late effect of treatment. The effects of adjuvant hormonal therapy on the brain and cognitive function, which are not addressed by this study, also need further evaluation.

\section{Answer d}

\section{Vascular safety in CML patients treated with second- and third-generation BCR-ABL1 TKIs ${ }^{4}$}

Imatinib, the first BCR-ABL1 tyrosine-kinase inhibitor (TKI) approved for the treatment of chronic myeloid leukemia (CML), was highly effective and had no significant vascular adverse events (VAEs) associated with its use. However the second- and third-generation agents have significant VAEs. This paper catalogues those VAEs and explores their etiologic mechanisms and possible management or even avoidance.

According to this review, arterial occlusive disease is most common in which TKIs:

a) Nilotinib and ponatinib

b) Imatinib and bosutinib

c) Dasatinib and bosutinib

d) All the TKIs approved for the treatment of CML

\section{Pleural effusion and pulmonary hypertension is} observed in patients treated with:
a) Nilotinib
b) Ponatinib
c) Bosutinib
d) Dasatinib

\section{Key points}

During the development of BCR-ABL1 tyrosine-kinase inhibitors for the treatment of CML, VAEs may have been overlooked for a number of reasons. Vascular disorders are common in elderly patients, in whom CML is more prevalent, and are often not considered as oncologic events during clinical trials. CML has not been directly connected to the etiology of atherosclerosis, and the initial clinical trials of CML therapies were not powered to detect VAEs.

Pleural effusion is recognized as an oncologic event, so the occurrence of pleural effusions during treatment with dasatinib was immediately recognized as an adverse event. By the time ponatinib was being developed, a potential relationship between TKI therapy and VAEs had been published.

Although occasionally reported in patients treated with imatinib, dasatinib, and bosutinib, arterial occlusive disease has most commonly been observed in patients on nilotinib and ponatinib.

The mechanisms of the vasculopathy caused by these drugs may include metabolic effects such as insulin resistance, pro-atherogenic and stenosis-augmenting effects such as direct inhibitory effects on vascular endothelial cells, and impairment of vascular repair after occlusion. There also may be effects on platelet function and mast cell depletion.

Management of the VAEs in patients with CML includes the elimination of all vascular risk factors in patients with low-grade vascular disease so treatment may be continued. For patients with higher-grade vascular disease, nilotinib or ponatinib may need to be replaced by another TKI or the drugs can be given for a short course of therapy to be 
followed by a TKI that has a lower risk for VAEs. Imatinib may still be the best choice in selected patients.

Answers a, d

\section{Predictors of heart failure in childhood cancer survivors ${ }^{5,6}$}

Heart disease is a late consequence of cancer therapy. This has been more frequently recognized in survivors of childhood cancer. There are now more than 400,000 such survivors in the United States and many are followed in adulthood by either medical oncologists or general internists, so reliable predictors of heart failure in this population of survivors would be very useful for these practitioners.

In the Childhood Cancer Survivor Study (CCSS), 13,060 survivors who had been free of cardiovascular disease at least 5 years after cancer diagnosis were observed through age 40 years for the development of CVD and their data was compared with data from 4,023 of their siblings. An additional 3,421 survivors from Emma Children's Hospital in Amsterdam and the US-based National Wilms Tumor Study and St Jude Lifetime Cohort Study were used for validation.

The strongest predictors of congestive heart failure in young cancer survivors included all of the following except:

a) Male sex

b) Younger age at cancer diagnosis

c) Increasing doses of anthracycline chemotherapy

d) Direct radiation to the chest or heart

\section{Key points}

Using the CCSS cohort, the investigators derived and tested a predictive model for the risk of heart failure in survivors of childhood cancer therapy. The discriminating factors were female gender, younger age at diagnosis, dose of anthracycline, and exposure to chest or cardiac radiation therapy. The incidence of congestive heart failure $(\mathrm{CHF})$ by age 40 was $0.5 \%, 2.4 \%$, and $11.7 \%$ for the low-, medium-, and high-risk groups of survivors, compared with $0.3 \%$ in the unaffected siblings.

The CCSS CHF prediction model provides a form of risk assessment for survivors of childhood cancer and its treatment. Use of such a model would allow for studies of screening and treatment interventions to lower $\mathrm{CHF}$ risk in the adult survivors of childhood cancers.

\section{Answer a}

\section{Risk of venous and arterial thrombotic events in patients diagnosed with SVT'}

Superficial vein thrombosis (SVT) is common, generally considered to be innocuous, and treated symptomati- cally. However, it has recently been more closely linked to deep venous thrombosis (DVT) and pulmonary embolism. These more dangerous forms of VTEs may occur synchronously or metachronously with an episode of SVT.

In this study of a large unselected population - the entire population of Denmark - 10,973 participants with SVT were compared with 515,067 participants matched by gender, age, and index date. The subsequent risks of DVT, pulmonary embolism, acute myocardial infarction, stroke, and death were analyzed and compared between the 2 groups.

\section{The primary outcome of this study was:}

a) The risk of DVT after SVT was highest after the initial 3 months of follow-up.

b) The incidence of DVT was about 11-fold higher in participants who had experienced an SVT than in those who had not experienced an SVT.

c) Most of the increase in DVTs after SVTs was in cancer patients.

d) DVT almost never occurred in the contralateral leg to the SVT.

e) $60 \%$ of SVT patients were women, and the relative risks of all thromboembolic outcomes were also higher in women.

\section{Key points}

SVT may not be as benign as was once thought. Current guidelines from the American College of Chest Physicians include a recommendation for 45 days of anticoagulation therapy for patients with SVT of at least $5 \mathrm{~cm}$ in length on the lower extremity. ${ }^{8}$ The results of 3 recent randomized trials including the CALISTO trial support this approach. ${ }^{9}$

In the large population-based, case-control Danish study, the risk of DVT was markedly increased among those who had an SVT compared with the general population, particularly during the initial 3 months after the SVT diagnosis. The relative risk was higher in men even though $60 \%$ of SVT patients were women. The hazard ratio was not changed after cancer patients were excluded from the analysis. DVT occurred in the contralateral leg in up to $10 \%$ of cases, indicating that superficial and deep venous thrombosis might result from a common hypercoaguable etiology.

\section{Answer b}

\section{VTE prophylaxis and treatment in patients with cancer: ASCO guidelines ${ }^{10,11}$}

ASCO published evidence-based practice guidelines for the treatment and prophylaxis of VTE in 2007, with an update published in 2013 (the 2012 update). The latest version for the 2014 update was published in February 2015 . 


\section{Feature}

The following recommendations are unchanged since the 2012 Update (include all that apply):

a) Most hospitalized patients with active cancer require thromboprophylaxis.

B) Patients with multiple myeloma receiving antiangiogenic agents with chemotherapy and/or dexamethasone should receive prophylaxis with either a low molecular weight heparin or aspirin.

c) Low molecular weight heparin is recommended for the initial 5-10 days of treatment for VTE, followed by an additional 6 months.

d) Novel oral anticoagulants should not be used in patients with malignancy and VTE.

e) VTE prophylaxis should be extended for 4 weeks after major abdominal or pelvic surgery with high-risk features.

\section{Key points}

The ASCO Update Committee reviewed the literature, including randomized clinical trials systematic reviews, meta-analyses, and other clinical practice guidelines, from November 5, 2012 through July 2014. After an analysis and review of this search, which included 53 publications, the committee concluded that there should be no changes in the 2012 guideline recommendations.

The highlights included a statement that routine prophylaxis is not recommended for ambulatory patients with cancer but could be considered for a highly selective group of high-risk patients. Anticoagulation should not be used to extend survival of patients with cancer. All patients should be periodically assessed for risk of VTE. Novel oral anticoagulants are still not recommended for patients with malignancy and VTE. Patients with multiple myeloma receiving antiangiogenic agents with chemotherapy and/or dexamethasone should receive prophylaxis. Most hospitalized patients with active cancer should receive prophylaxis as should patients undergoing major cancer surgery. The latter patients should receive prophylaxis before surgery as well as for at least 7-10 days afterward. Extended prophylaxis should be given to patients who are undergoing major abdominal or pelvic surgery with high-risk features.

\section{Answers a, b, c, d, e}

\section{References}

1. O'Farrell S, Garmo H, Holmberg L, et al. Risk and timing of cardiovascular disease after androgen - deprivation therapy in men with prostate cancer. J Clin Oncol. 2015;33:1243-1251.

2. 'Basaria S. cardiovascular disease associated with androgen - deprivation therapy: time to give it due respect. J Clin Oncol. 2015;33:1232-1234.

3. Koppelmans V, Vernooij MW, BoogerdW, et al. Prevalence of cerebral small-vessel disease in long-term breast cancer survivors exposed to both adjuvant radiotherapy and chemotherapy. J Clin Oncol. 32015;3:588-593.

4. Valent $\mathrm{P}$, Hadzijusufovic E, Schernthaner G-H, et al. Vascular safety issues in CML patients treated with BCR-ABL1 kinase inhibitors. Blood. 2015;125:901-906

5. Chow EJ, Chen Y, Kremer LC, et al. Individual prediction of heart failure among childhood cancer survivors. J Clin Oncol. 2015;33:394-402.

6. Bluhm EC, Barac A. Heart failure risk reduction in childhood cancer survivors: where is our crystal ball? J Clin Oncol. 2015;33:379-380.

7. Cannegieter SC, Horvath-Puno E, Schmidt M, et al. Risk of venous and arterial thrombotic events in patients diagnosed with superficial vein thrombosis: a nationwide cohort study. Blood. 2015;125:229235.

8. Kearon C, Aki EA, Comerota AJ, et al. American College of Chest Physicians. Antithrombotic therapy for VTE disease: anithrombotic therapy and prevention of thrombosis, 9th ed: American College of Chest Physicians evidence-based clinical practice guidelines [published correction in Chest. 2012 Dec;142(6):1698-1704]. Chest. 2012; 41(2 suppl): 3419S-494S.

9. Decousus H, Prandoni P, Mismetti P, et al, for the CALISTO Study Group. Fondaparinux for the treatment of superficial-vein thrombosis in the legs. N Engl J Med. 2010;363:1222-1232.

10. Lyman, GH, Khorana AA, Kuderer NM, et al. Venous thromboembolism prophylaxis and treatment in patients with cancer: American Society of Clinical Oncology clinical practice guideline update. J Clin Oncol. 2013;31:2189-2204.

11. Lyman GH, Bohlke K, Khorana AA, et al. Venous thromboembolism prophylaxis and treatment in patients with cancer: American Society of Clinical Oncology clinical practice guideline update 2014.. J Clin Oncol. 2015;33:654-656. 